\title{
AN EXTENSION OF TYCHONOFF FIXED POINT THEOREM WITH APPLICATION TO THE SOLVABILITY OF THE INFINITE SYSTEMS OF INTEGRAL EQUATIONS IN THE FRÉCHET SPACES
}

\author{
REZA ALLAHYARI, REZA ARAB, AND ALI SHOLE HAGHIGHI
}

Received 30 May, 2015

\begin{abstract}
In the present article, we introduce a new concept of contraction and prove a new type of the extension of Tychonoff fixed point theorem. Then, as an application, we study the problem of existence of solutions for the infinite systems of integral equations using the technique of measures of noncompactness in conjunction with this extension in the Fréchet spaces.
\end{abstract}

2010 Mathematics Subject Classification: $54 \mathrm{H} 25 ; 47 \mathrm{H} 10$

Keywords: measure of noncompactness, Tychonoff fixed point theorem, infinite systems of integral equations

\section{INTRODUCTION}

The theory of infinite systems of integral or differential equations creates an important branch of nonlinear analysis. Up to now, several papers have been published on this significant topic (see $[9,12,16,17,19,21]$ ) . It is connected naturally with a large number of problems considered in mechanics, engineering, in the theory of branching processes, the theory of neutral nets and so on (see [14, 15, 18, 20, 22, 23]). In this paper, we investigate the existence of solutions for the infinite systems of integral equations of the forms

$$
\begin{aligned}
x_{n}(t)= & f_{n}\left(t, x_{1}(t), \ldots, x_{n}(t)\right) \\
& +q_{n}\left(t, x_{1}(t), \ldots, x_{n}(t)\right) \int_{0}^{\beta_{n}(t)} g_{n}\left(t, s,\left(x_{j}(s)\right)_{j=1}^{\infty}\right) d s, n \in \mathbb{N}, t \in \mathbb{R},
\end{aligned}
$$

and

$$
\begin{aligned}
y_{n}(t)= & f_{n}\left(t, y_{1}(t), \ldots, y_{n}(t)\right) \\
& +q_{n}\left(t, y_{1}(t), \ldots, y_{n}(t)\right) \int_{0}^{\infty} k_{n}(t, s) h_{n}\left(s,\left(y_{j}(s)\right)_{j=1}^{\infty}\right) d s, n \in \mathbb{N}, t \in \mathbb{R},
\end{aligned}
$$

by using the measures of noncompactness $\mu$ introduced in [9] and a new type of extension of Tychonoff fixed point theorem in the Fréchet space $\left(B C\left(\mathbb{R}_{+}\right)\right)^{\omega}$ (denote 
the countable cartesian product of $B C\left(\mathbb{R}_{+}\right)$with itself). The results of this paper improve and generalize those obtained in papers $[2-8,10,11]$.

\section{NOTATION AND AUXILIARY FACTS}

Here, we recall some basic facts concerning measures of noncompactness. Denote by $\mathbb{R}$ the set of real numbers and put $\mathbb{R}_{+}=[0,+\infty)$. The symbol $\bar{X}$ and $\operatorname{Conv} X$ will denote the closure and closed convex hull of a subset $X$ of $E$, respectively. Moreover, let $\mathfrak{N}_{E}$ indicate the family of all nonempty and relatively compact subsets of $E$.

A topological vector space (TVS) is a vector space $X$ over the field $\mathbb{R}$ which is endowed with a topology such that the maps $(x, y) \rightarrow x+y$ and $(\alpha, x) \rightarrow \alpha x$ are continuous from $X \times X$ and $\mathbb{R} \times X$ to $X$. A topological vector space is called locally convex if there is a basis for the topology consisting of convex sets (that is, set $A$ such that if $x, y \in A$ then $t x+(1-t) y \in A$ for $0<t<1)$.

Definition 1 ([13]). A Fréchet space is a locally convex space which is complete with respect to a translation-invariant metric.

Example 1. Let $E_{i}$ be a Banach space for all $i \in \mathbb{N}$, then $\prod_{i \in \mathbb{N}} E_{i}$ is a Fréchet space with respect to the metric

$$
\begin{aligned}
& \qquad d(x, y)=\sup \left\{\frac{1}{2^{i}} \min \left\{1, d_{i}\left(x_{i}, y_{i}\right)\right\}: i \in \mathbb{N}\right\}, \\
& \text { where } x=\left(x_{1}, x_{2}, \ldots\right), y=\left(y_{1}, y_{2}, \ldots\right) \in \prod_{i \in \mathbb{N}} E_{i} .
\end{aligned}
$$

Definition 2 ([9]). Let $\mathcal{M}$ be a class of subsets of a Fréchet space E, we say $\mathcal{M}$ is admissible class if $\mathfrak{N}_{E} \cap \mathcal{M} \neq \varnothing$ and if $X \in \mathcal{M}$, then $\operatorname{Conv}(X), \bar{X} \in \mathcal{M}$.

Definition 3 ([9]). Let $\mathcal{M}$ be an admissible class of a Fréchet space E, we say that $\mu: \mathcal{M} \longrightarrow \mathbb{R}_{+}$is a measure of noncompactness on Fréchet space $\mathrm{E}$ if it satisfies the following conditions:

$\left(1^{\circ}\right)$ The family $\operatorname{ker} \mu=\{X \in \mathcal{M}: \mu(X)=0\}$ is nonempty and $\operatorname{ker} \mu \subseteq \mathfrak{N}_{E}$;

$\left(2^{\circ}\right) X \subset Y \Longrightarrow \mu(X) \leq \mu(Y)$;

$\left(3^{\circ}\right) \mu(\bar{X})=\mu(X)$

(4) $\mu(\operatorname{Conv} X)=\mu(X)$;

$\left(5^{\circ}\right) \mu(\lambda X+(1-\lambda) Y) \leq \lambda \mu(X)+(1-\lambda) \mu(Y)$ for $\lambda \in[0,1]$;

$\left(6^{\circ}\right)$ If $\left\{X_{n}\right\}$ is a sequence of closed sets from $\mathcal{M}$ such that $X_{n+1} \subset X_{n}$ for $n=$ $1,2, \cdots$, and if $\lim _{n \rightarrow \infty} \mu\left(X_{n}\right)=0$, then $X_{\infty}=\cap_{n=1}^{\infty} X_{n} \neq \varnothing$.

Theorem 1 (Tychonoff fixed point theorem [1]). Let E be a Hausdorff locally convex linear topological space, $C$ be a convex subset of $E$ and $F: C \longrightarrow E$ be a continuous mapping such that

$$
F(C) \subseteq A \subseteq C,
$$


with A compact. Then $F$ has at least one fixed point.

Theorem 2 ([9]). Suppose $\mu_{i}$ be a measure of noncompactness on Banach spaces $E_{i}$ for all $i \in \mathbb{N}$. If we define

$$
\mathcal{M}=\left\{C \subseteq \prod_{i=1}^{\infty} E_{i}: \sup _{i}\left\{\mu_{i}\left(\pi_{i}(C)\right)\right\}<\infty\right\},
$$

where $\pi_{i}(C)$ denotes the natural projection of $\prod_{i=1}^{\infty} E_{i}$ into $E_{i}$ and $\mu: \mathcal{M} \longrightarrow \mathbb{R}_{+}$is defined by

$$
\mu(C)=\sup \left\{\mu_{i}\left(\pi_{i}(C)\right): i \in \mathbb{N}\right\}
$$

then $\mathcal{M}$ is an admissible set and $\mu$ is a measure of noncompactness on $X=\prod_{i=1}^{\infty} E_{i}$.

\section{MAIN RESULT}

In this section, we introduce a new concept of contraction and prove some new extensions of Tychonoff fixed point theorem.

Theorem 3. Let $\Omega$ be a nonempty, closed and convex subset of a Fréchet space $E$, $\mathcal{M}$ is admissible class such that $\Omega \in \mathcal{M}$ and $\mu: \mathcal{M} \longrightarrow \mathbb{R}_{+}$is a measure of noncompactness on $E$. Let $F, G: \Omega \longrightarrow \Omega$ be two continuous mappings such that

$$
\mu(F X)+\psi(\mu(G Y)) \leq \varphi(\mu(X)+\psi(\mu(Y))),
$$

and $F(X), G(Y) \in \mathcal{M}$ for any nonempty subset $X, Y \in \mathcal{M}$ where $\varphi, \psi: \mathbb{R}_{+} \longrightarrow \mathbb{R}_{+}$ are nondecreasing and right continuous functions such that $\varphi(0)=\psi(0)=0$ and $\varphi(t)<t$ for each $t>0$. Then $F$ and $G$ have at least one fixed point in the set $\Omega$.

Proof. By induction, we obtain sequences $\left\{\Omega_{n}\right\}$ and $\left\{\Lambda_{n}\right\}$ such that

$$
\left\{\begin{array}{ll}
\Omega_{0}=\Lambda_{0}=\Omega, & \\
\Omega_{n}=\operatorname{Conv}\left(F \Omega_{n-1}\right) & n \geq 1, \quad \\
\Lambda_{n}=\operatorname{Conv}\left(G \Lambda_{n-1}\right) & n \geq 1 .
\end{array} .\right.
$$

It is obvious that $\Omega_{n}, \Lambda_{n} \in \mathcal{M}$ for all $n \in \mathbb{N}$. If there exists an integer $N \geq 0$ such that $\mu\left(\Omega_{N}\right)=\mu\left(\Lambda_{N}\right)=0$, then $\Omega_{N}$ and $\Lambda_{N}$ are compact. Thus, Theorem 1 implies that $F$ and $G$ have a fixed point. Now assume that $\mu\left(\Omega_{n}\right) \neq 0$ or $\mu\left(\Lambda_{n}\right) \neq 0$ for $n \geq 0$. Since we have $F \Omega_{0}=F \Omega \subseteq \Omega=\Omega_{0}, \Omega_{1}=\operatorname{Conv}\left(F \Omega_{0}\right) \subseteq \Omega=\Omega_{0}$, and by continuing this process we obtain

$$
\Omega_{0} \supseteq \Omega_{1} \supseteq \Omega_{2} \supseteq \cdots
$$

and

$$
\Lambda_{0} \supseteq \Lambda_{1} \supseteq \Lambda_{2} \supseteq \cdots,
$$


so $\mu\left(\Omega_{n}\right)$ and $\mu\left(\Lambda_{n}\right)$ is a positive decreasing sequence of real numbers. Thus, there are $r_{1}, r_{2} \geq 0$ such that $\mu\left(\Omega_{n}\right) \longrightarrow r_{1}$ and $\mu\left(\Lambda_{n}\right) \longrightarrow r_{2}$ as $n \longrightarrow \infty$. On the other hand, in view of (3.1), we obtain

$$
\limsup _{n \longrightarrow \infty} \mu\left(\Omega_{n+1}\right)+\psi\left(\mu\left(\Lambda_{n+1}\right) \leq \limsup _{n \longrightarrow \infty} \varphi\left(\mu\left(\Omega_{n}\right)+\psi\left(\mu\left(\Lambda_{n}\right)\right)\right) .\right.
$$

This show that $r_{1}+\psi\left(r_{2}\right) \leq \varphi\left(r_{1}+\psi\left(r_{2}\right)\right)$. Consequently $r_{1}+\psi\left(r_{2}\right)=0$, so $r_{1}=$ $r_{2}=0$. Hence we deduce that $\mu\left(\Omega_{n}\right) \longrightarrow 0$ and $\mu\left(\Lambda_{n}\right) \longrightarrow 0$ as $n \longrightarrow \infty$. Since the sequences $\left(\Omega_{n}\right)$ and $\left(\Lambda_{n}\right)$ are nested, in view of axiom $\left(6^{\circ}\right)$ of Definition 3 we derive that the sets $\Omega_{\infty}=\bigcap_{n=1}^{\infty} \Omega_{n}$ and $\Lambda_{\infty}=\bigcap_{n=1}^{\infty} \Lambda_{n}$ are nonempty, closed and convex subsets of the set $\Omega$. Moreover, the sets $\Omega_{\infty}$ and $\Lambda_{\infty}$ are invariant under the operators $F$ and $G$ respectively, and belongs to $\operatorname{ker} \mu$. Now, Tychonoff fixed point theorem implies that $F$ and $G$ have fixed points in the set $\Omega$.

Corollary 1. Let $\Omega$ be a nonempty, closed and convex subset of a Fréchet space $E, \mathcal{M}$ satisfies the hypotheses of Theorem 3 and $\Omega \in \mathcal{M}$. Let $F, G: \Omega \longrightarrow \Omega$ be two continuous mappings such that

$$
\mu(F X)+\psi(\mu(G Y)) \leq k[\mu(X)+\psi(\mu(Y))],
$$

and $F(X), G(Y) \in \mathcal{M}$ for any nonempty subset $X, Y \in \mathcal{M}$ where $\psi: \mathbb{R}_{+} \longrightarrow \mathbb{R}_{+}$is nondecreasing and right continuous function such that $\psi(0)=0, \mu$ is an arbitrary measure of noncompactness on $\mathcal{M}$ and $k \in[0,1)$. Then $F$ and $G$ have at least a fixed point in the set $\Omega$.

Proof. Take $\varphi(t)=k t$ in Theorem 3 .

Definition 4. Let $X$ be a Banach space. An operator $F: X \longrightarrow X$ is affine if $F(a x+(1-a) y)=a F(x)+(1-a) F(y)$ for all $x, y \in X$ and all $a \in \mathbb{R}$.

Corollary 2. Let $\Omega$ be a nonempty, closed and convex subset of a Fréchet space $E, \mathcal{M}$ is admissible class such that $\Omega \in \mathcal{M}$ and $\mu: \mathcal{M} \longrightarrow \mathbb{R}_{+}$is a measure of noncompactness on $E$. Let $F, G: \Omega \longrightarrow \Omega$ be two continuous operators such that

$$
\mu(F X) \leq \varphi(\mu(X))
$$

and $F(X) \in \mathcal{M}$ for any nonempty subset $X \in \mathcal{M}$ where $\varphi: \mathbb{R}_{+} \longrightarrow \mathbb{R}_{+}$satisfies the hypotheses of Theorem 3. Moreover, $G(F(X)) \subseteq F(X)$ for all $X \in \mathfrak{N}_{\Omega}$ and $F$ is affine operator. Then $F$ and $G$ have at least one common fixed point in the set $\Omega$.

Proof. Take $\psi(t)=0$ in Theorem 3. Thus, applying Theorem 3, $F$ has a fixed point. Now suppose that $\Gamma=\{x \in \Omega: F x=x\}$, then by (3.2) and $F(\Gamma)=\Gamma$ we have

$$
\begin{aligned}
\mu(\Gamma) & =\mu(F(\Gamma)) \\
& \leq \varphi(\mu(\Gamma)) \\
& <\mu(\Gamma) .
\end{aligned}
$$


which is a contradiction. So $\Gamma$ is relatively compact, and since $F$ is a continuous and affine operator so $\Gamma$ is nonempty, compact and convex subset of $\Omega$. Also, we have

$$
\begin{aligned}
G(\Gamma) & =G(F(\Gamma)) \\
& \subseteq F(\Gamma) \\
& =\Gamma .
\end{aligned}
$$

Now, Tychonoff fixed point theorem implies that $F$ and $G$ have a common fixed point in the set $\Gamma$.

We introduce the following useful corollary which will be used in Section 4.

Corollary 3. Let $\Omega_{i}(i \in \mathbb{N})$ be a nonempty, convex and closed subset of a Banach space $E_{i}, \mu_{i}$ an arbitrary measure of noncompactness on $E_{i}$ and $\sup _{i}\left\{\mu_{i}\left(\Omega_{i}\right)\right\}<\infty$. Let $F_{i}, G_{i}: \prod_{i=1}^{\infty} \Omega_{i} \longrightarrow \Omega_{i}(i=1,2, \ldots)$ be continuous operators such that

$$
\mu_{i}\left(F_{i}\left(\prod_{i=1}^{\infty} X_{i}\right)\right)+\psi\left(\mu_{i}\left(G_{i}\left(\prod_{i=1}^{\infty} Y_{i}\right)\right)\right) \leq \varphi\left(\sup _{i}\left\{\mu_{i}\left(X_{i}\right)+\psi\left(\mu_{i}\left(Y_{i}\right)\right)\right\}\right)
$$

for any subsets $X_{i}$ and $Y_{i}$ of $\Omega_{i}(i \in \mathbb{N})$ where $\varphi, \psi: \mathbb{R}_{+} \longrightarrow \mathbb{R}_{+}$satisfies the hypotheses of Theorem 3. Then there exist $\left(x_{j}^{*}\right)_{j=1}^{\infty},\left(y_{j}^{*}\right)_{j=1}^{\infty} \in \prod_{j=1}^{\infty} \Omega_{j}$ such that for all $i \in \mathbb{N}$

$$
\left\{\begin{array}{l}
F_{i}\left(\left(x_{j}^{*}\right)_{j=1}^{\infty}\right)=x_{i}^{*}, \\
G_{i}\left(\left(y_{j}^{*}\right)_{j=1}^{\infty}\right)=y_{i}^{*} .
\end{array}\right.
$$

Proof. Assume that $\widetilde{F}, \widetilde{G}: \prod_{i=1}^{\infty} \Omega_{i} \longrightarrow \prod_{i=1}^{\infty} \Omega_{i}$ are defined as follows

$$
\widetilde{F}\left(\left(x_{j}\right)_{j=1}^{\infty}\right)=\left(F_{1}\left(\left(x_{j}\right)_{j=1}^{\infty}\right), F_{2}\left(\left(x_{j}\right)_{j=1}^{\infty}\right), \ldots, F_{i}\left(\left(x_{j}\right)_{j=1}^{\infty}\right), \ldots\right),
$$

and

$$
\widetilde{G}\left(\left(y_{j}\right)_{j=1}^{\infty}\right)=\left(G_{1}\left(\left(y_{j}\right)_{j=1}^{\infty}\right), G_{2}\left(\left(y_{j}\right)_{j=1}^{\infty}\right), \ldots, G_{i}\left(\left(y_{j}\right)_{j=1}^{\infty}\right), \ldots\right)
$$

for all $\left(x_{j}\right)_{j=1}^{\infty},\left(y_{j}\right)_{j=1}^{\infty} \in \prod_{i=1}^{\infty} \Omega_{i}$. It is obvious that $F$ and $G$ are continuous. It suffices to show that the hypothesis (3.1) of Theorem 3 holds where $\mu$ is defined by Theorem 2. Take arbitrary nonempty subset $X$ and $Y$ of $\prod_{i=1}^{\infty} \Omega_{i}$. Now, by $\left(2^{\circ}\right)$ and (3.3) we 
obtain

$$
\begin{aligned}
\mu(\widetilde{F}(X))+\psi(\mu(\widetilde{G}(Y))) & \leq \mu\left(\prod_{i=1}^{\infty} F_{i}\left(\prod_{j=1}^{\infty} \pi_{j}(X)\right)\right)+\psi\left(\mu\left(\prod_{i=1}^{\infty} G_{i}\left(\prod_{j=1}^{\infty} \pi_{j}(Y)\right)\right)\right) \\
& =\sup _{i} \mu_{i}\left(F_{i}\left(\left(\prod_{j=1}^{\infty} \pi_{j}(X)\right)\right)\right)+\psi\left(\sup _{i} \mu_{i}\left(G_{i}\left(\left(\prod_{j=1}^{\infty} \pi_{j}(Y)\right)\right)\right)\right) \\
& \leq \sup _{i}\left\{\mu_{i}\left(F_{i}\left(\left(\prod_{j=1}^{\infty} \pi_{j}(X)\right)\right)\right)+\psi\left(\mu_{i}\left(G_{i}\left(\left(\prod_{j=1}^{\infty} \pi_{j}(Y)\right)\right)\right)\right)\right\} \\
& \leq \sup _{i} \varphi\left(\sup _{j}\left\{\mu_{j}\left(X_{j}\right)+\psi\left(\mu_{j}\left(Y_{j}\right)\right)\right\}\right) \\
& \leq \varphi\left(\sup _{j} \mu_{j}\left(X_{j}\right)+\psi\left(\sup _{j} \mu_{j}\left(Y_{j}\right)\right)\right) \\
& \leq \varphi(\mu(X)+\psi(\mu(Y))) .
\end{aligned}
$$

Therefore, all the conditions of Theorem 3 are satisfied, hence $\widetilde{F}$ and $G$ have fixed points and there exist $\left(x_{j}^{*}\right)_{j=1}^{\infty},\left(y_{j}^{*}\right)_{j=1}^{\infty} \in \prod_{j=1}^{\infty} \Omega_{j}$ such that

$$
\left\{\begin{array}{c}
\left(x_{j}^{*}\right)_{j=1}^{\infty}=\widetilde{F}\left(\left(x_{j}^{*}\right)_{j=1}^{\infty}\right)=\left(F_{1}\left(\left(x_{j}^{*}\right)_{j=1}^{\infty}\right), F_{2}\left(\left(x_{j}^{*}\right)_{j=1}^{\infty}\right), \ldots, F_{j}\left(\left(x_{j}^{*}\right)_{j=1}^{\infty}\right), \ldots\right), \\
\left(y_{j}^{*}\right)_{j=1}^{\infty}=\widetilde{G}\left(\left(y_{j}^{*}\right)_{j=1}^{\infty}\right)=\left(G_{1}\left(\left(x_{j}^{*}\right)_{j=1}^{\infty}\right), G_{2}\left(\left(x_{j}^{*}\right)_{j=1}^{\infty}\right), \ldots, G_{j}\left(\left(x_{j}^{*}\right)_{j=1}^{\infty}\right), \ldots\right),
\end{array}\right.
$$

and that (3.4) holds.

\section{APPLICATION}

In this section we are going to show how the measure of noncompactness defined in the previous section, can be applied to an infinite system of nonlinear integral equations. Here, we will work in the classical Banach space $B C\left(\mathbb{R}_{+}\right)$consisting of all real functions defined, bounded and continuous on $\mathbb{R}_{+}$equipped with the standard norm

$$
\|x\|=\sup \{|x(t)|: t \geq 0\} .
$$

Now, we present the definition of a special measure of noncompactness in $B C\left(\mathbb{R}_{+}\right)$ which will be needed in the sequel.

To do this, let $X$ be a fixed nonempty and bounded subset of $B C\left(\mathbb{R}_{+}\right)$and fix a positive number $T$. For $x \in X$ and $\varepsilon>0$, denote by $\omega^{T}(x, \varepsilon)$ the modulus of the continuity of function $x$ on the interval $[0, T]$, i.e.,

$$
\omega^{T}(x, \varepsilon)=\sup \{|x(t)-x(s)|: t, s \in[0, T],|t-s| \leq \varepsilon\} .
$$

Further, let us put

$$
\begin{gathered}
\omega^{T}(X, \varepsilon)=\sup \left\{\omega^{T}(x, \varepsilon): x \in X\right\}, \\
\omega_{0}^{T}(X)=\lim _{\varepsilon \rightarrow 0} \omega^{T}(X, \varepsilon)
\end{gathered}
$$


and

$$
\omega_{0}(X)=\lim _{T \rightarrow \infty} \omega_{0}^{T}(X) .
$$

If $\mathrm{t}$ is a fixed number from $\mathbb{R}_{+}$, let us denote $X(t)=\{x(t): x \in X\}$. Finally, consider the function $\mu$ defined on $\mathfrak{M}_{B C\left(\mathbb{R}_{+}\right)}$by the formula

$$
\mu(X)=\omega\left(X_{0}\right)+\limsup _{t \rightarrow \infty} \operatorname{diam} X(t),
$$

where,

$$
\operatorname{diam} X(t)=\sup \{|x(t)-y(t)|: x, y \in X\} .
$$

It is shown [10] that the function $\mu$ is a measure of noncompactness in the space $B C\left(\mathbb{R}_{+}\right)$.

We will consider Equations (1.1) and (1.2) under the following assumptions:

$\left(A_{1}\right) f_{n}, q_{n}: \mathbb{R}_{+} \times \mathbb{R}^{n} \longrightarrow \mathbb{R}(n \in \mathbb{N})$ are continuous with

$$
M:=\sup \left\{\max \left\{\left|f_{n}(t, 0, \ldots, 0)\right|,\left|q_{n}(t, 0, \ldots, 0)\right|\right\}: t \in \mathbb{R}_{+}, n \in \mathbb{N}\right\}<\infty .
$$

Moreover, there exists a nondecreasing, concave and upper semicontinuous function $\varphi$ with $\varphi(t)<t$ for all $t>0$ such that

$$
\left|f_{n}\left(t, x_{1}, \ldots, x_{n}\right)-f_{n}\left(t, y_{1}, \ldots, y_{n}\right)\right| \leq \varphi\left(\max _{1 \leq i \leq n}\left|x_{i}-y_{i}\right|\right),
$$

and

$$
\left|q_{n}\left(t, x_{1}, \ldots, x_{n}\right)-q_{n}\left(t, y_{1}, \ldots, y_{n}\right)\right| \leq \varphi\left(\max _{1 \leq i \leq n}\left|x_{i}-y_{i}\right|\right) ;
$$

$\left(B_{1}\right) \beta_{n}: \mathbb{R}_{+} \longrightarrow[0, \infty)$ is continuous function for all $n \in \mathbb{N}$;

$\left(\mathrm{B}_{2}\right) g_{n}: \mathbb{R}_{+} \times \mathbb{R}_{+} \times \mathbb{R}^{\omega} \longrightarrow \mathbb{R}(n \in \mathbb{N})$ is continuous and there exists a positive constant $D$ such that

$D:=\sup \left\{\left|\int_{0}^{\beta_{n}(t)} g_{n}\left(t, s,\left(x_{j}(s)\right)_{j=1}^{\infty}\right) d s\right|: t \in \mathbb{R}_{+}, x_{j} \in B C\left(\mathbb{R}_{+}\right), 1 \leq n<\infty\right\}$.

Moreover,

$$
\lim _{t \longrightarrow \infty}\left|\int_{0}^{\beta_{n}(t)} g_{n}\left(t, s,\left(x_{j}(s)\right)_{j=1}^{\infty}\right)-g_{n}\left(t, s,\left(y_{j}(s)\right)_{j=1}^{\infty}\right) d s\right|=0,
$$

uniformly respect to $x_{j}, y_{j} \in B C\left(\mathbb{R}_{+}\right)$;

$\left(C_{1}\right) k_{n}: \mathbb{R}_{+} \times \mathbb{R}_{+} \longrightarrow \mathbb{R}$ is continuous function for all $n \in \mathbb{N}$;

$\left(C_{2}\right) h_{n}: \mathbb{R}_{+} \times \mathbb{R}^{\omega} \longrightarrow \mathbb{R}(n \in \mathbb{N})$ is a continuous and there exists a continuous function $a_{n}: \mathbb{R}_{+} \longrightarrow \mathbb{R}_{+}$and a continuous and nondecreasing function $b_{n}$ : $\mathbb{R}_{+} \longrightarrow \mathbb{R}_{+}$such that

$$
\left|h_{n}\left(t,\left(x_{j}\right)_{j=1}^{\infty}\right)\right| \leq a_{n}(t) b_{n}\left(\sup _{1 \leq j<\infty}\left|x_{j}\right|\right),
$$

for all $t \in \mathbb{R}_{+}$and $\left(x_{j}\right)_{j=1}^{\infty} \in \mathbb{R}^{\omega}$ with $\sup _{1 \leq j<\infty}\left|x_{j}\right|<\infty$. Also the function $s \longrightarrow$ $a_{n}(s) k_{n}(t, s)$ is integrable over $\mathbb{R}_{+}$for any fixed $t \in \mathbb{R}_{+}$and $n \in \mathbb{N}$; 
$\left(C_{3}\right)$ There exists a positive constant $Q$ such that

$$
Q:=\sup \left\{\int_{0}^{\infty} a_{n}(s)\left|k_{n}(t, s)\right| d s: t \in \mathbb{R}_{+}, n \in \mathbb{N}\right\}<\infty
$$

and

$$
\lim _{t \rightarrow \infty} \int_{0}^{\infty} a_{n}(s)\left|k_{n}(t, s)\right| d s=0 .
$$

$\left(C_{4}\right)$ The following equality holds:

$$
\lim _{T \rightarrow \infty}\left\{\sup \left\{\int_{T}^{\infty} a_{n}(s)\left|k_{n}(t, s)\right| d s: t \in \mathbb{R}_{+}\right\}\right\}=0
$$

for all $n \in \mathbb{N}$;

$\left(D_{1}\right)$ There exists a positive solution $r_{0}$ of the inequalities

$$
(1+D)(\varphi(r)+M) \leq r
$$

and

$$
\left(1+Q b_{n}(r)\right)(\varphi(r)+M) \leq r
$$

for all $n \in \mathbb{N}$ such that

$$
\left(\max \left\{1+D, \sup _{n \in \mathbb{N}}\left\{1+Q b_{n}\left(r_{0}\right)\right\}\right\}\right) \varphi(t)<t .
$$

Theorem 4. Under above conditions Eq. (1.1) and Eq. (1.2) have at least one solution in the space $\left(B C\left(\mathbb{R}_{+}\right)\right)^{\omega}$.

Proof. Let us fix arbitrarily $n \in \mathbb{N} . F_{n}, G_{n}:\left(B C\left(\mathbb{R}_{+}\right)\right)^{\omega} \longrightarrow B C\left(\mathbb{R}_{+}\right)(n \in \mathbb{N})$ are defined by

$$
\begin{aligned}
F_{n}\left(\left(x_{j}\right)_{j=1}^{\infty}\right)(t)= & f_{n}\left(t, x_{1}(t), \ldots, x_{n}(t)\right) \\
& +q_{n}\left(t, x_{1}(t), \ldots, x_{n}(t)\right) \int_{0}^{\beta_{n}(t)} g_{n}\left(t, s,\left(x_{j}(s)\right)_{j=1}^{\infty}\right) d s,
\end{aligned}
$$

and

$$
\begin{aligned}
G_{n}\left(\left(x_{j}\right)_{j=1}^{\infty}\right)(t)= & f_{n}\left(t, x_{1}(t), \ldots, x_{n}(t)\right) \\
& +q_{n}\left(t, x_{1}(t), \ldots, x_{n}(t)\right) \int_{0}^{\infty} k_{n}(t, s) h_{n}\left(s,\left(x_{j}(s)\right)_{j=1}^{\infty}\right) d s .
\end{aligned}
$$


In view of imposed assumptions, we infer that the operators $F_{n}\left(\left(x_{j}\right)_{j=1}^{\infty}\right)$ and $G_{n}\left(\left(x_{j}\right)_{j=1}^{\infty}\right)$ are continuous for arbitrarily $\left(x_{j}\right)_{j=1}^{\infty} \in\left(B C\left(\mathbb{R}_{+}\right)\right)^{\omega}$. Also from our assumptions, we obtain

$$
\begin{aligned}
& \left|F_{n}\left(\left(x_{j}\right)_{j=1}^{\infty}\right)(t)\right| \leq\left|f_{n}\left(t, x_{1}(t), \ldots, x_{n}(t)\right)\right| \\
& \quad+\left|q_{n}\left(t, x_{1}(t), \ldots, x_{n}(t)\right)\right|\left|\int_{0}^{\beta_{n}(t)} g_{n}\left(t, s,\left(x_{j}(s)\right)_{j=1}^{\infty}\right) d s\right| \\
& \leq\left|f_{n}\left(t, x_{1}(t), \ldots, x_{n}(t)\right)-f_{n}(t, 0, \ldots, 0)\right|+\left|f_{n}(t, 0, \ldots, 0)\right| \\
& \quad+\left(\left|q_{n}\left(t, x_{1}(t), \ldots, x_{n}(t)\right)-q_{n}(t, 0, \ldots, 0)\right|+\left|q_{n}(t, 0, \ldots, 0)\right|\right) \\
& \quad \cdot\left|\int_{0}^{\beta_{n}(t)} g_{n}\left(t, s,\left(x_{j}(s)\right)_{j=1}^{\infty}\right) d s\right| \\
& \leq \varphi\left(\max _{1 \leq i \leq n}\left|x_{i}(t)\right|\right)+M+D\left(\varphi\left(\max _{1 \leq i \leq n}\left|x_{i}(t)\right|\right)+M\right) \\
& \leq(1+D)\left(\varphi\left(\max _{1 \leq i \leq n}\left|x_{i}(t)\right|\right)+M\right) .
\end{aligned}
$$

Thus,

$$
\left\|F_{n}\left(\left(x_{j}\right)_{j=1}^{\infty}\right)\right\| \leq(1+D)\left(\varphi\left(\max _{1 \leq i \leq n}\left\|x_{i}\right\|\right)+M\right),
$$

and with similar argument

$$
\left\|G_{n}\left(\left(x_{j}\right)_{j=1}^{\infty}\right)\right\| \leq\left(1+Q b_{n}\left(\sup _{1 \leq j<\infty}\left\|x_{j}\right\|\right)\right)\left(\varphi\left(\max _{1 \leq i \leq n}\left\|x_{i}\right\|\right)+M\right) .
$$

$F_{n}\left(\left(x_{j}\right)_{j=1}^{\infty}\right)$ and $G_{n}\left(\left(x_{j}\right)_{j=1}^{\infty}\right) \in B C\left(\mathbb{R}_{+}\right)$for any $\left(x_{j}\right)_{j=1}^{\infty} \in\left(B C\left(\mathbb{R}_{+}\right)\right)^{\omega}$ with $\sup _{1 \leq j<\infty}\left\|x_{j}\right\|<\infty$. Due to Inequalities (4.1), (4.2) and using $\left(D_{1}\right)$, the opretors $F_{n}$ and $G_{n}$ maps $\left(\bar{B}_{r_{0}}\right)^{\omega}$ into $\bar{B}_{r_{0}}$. Now we show that $G_{n}$ is a continuous function on $\left(\bar{B}_{r_{0}}\right)^{\omega}$. To prove this, let us fix $0<\varepsilon<\frac{1}{2^{n}}$ and take arbitrary $x=\left(x_{j}\right)_{j=1}^{\infty}, y=$ $\left(y_{j}\right)_{j=1}^{\infty} \in\left(\bar{B}_{r_{0}}\right)^{\omega}$ such that $d(x, y)=\sup \left\{\frac{1}{2^{i}} \min \left\{1,\left\|x_{i}-y_{i}\right\|\right\}: i \in \mathbb{N}\right\}<\varepsilon$. Then, for $t \in \mathbb{R}_{+}$, we have 


$$
\begin{aligned}
& \left|G_{n}\left(\left(x_{j}\right)_{j=1}^{\infty}\right)(t)-G_{n}\left(\left(y_{j}\right)_{j=1}^{\infty}\right)(t)\right| \\
& =\mid f_{n}\left(t, x_{1}(t), \ldots, x_{n}(t)\right)+q_{n}\left(t, x_{1}(t), \ldots, x_{n}(t)\right) \int_{0}^{\infty} k_{n}(t, s) h_{n}\left(s,\left(x_{j}(s)\right)_{j=1}^{\infty}\right) d s \\
& -f_{n}\left(t, y_{1}(t), \ldots, y_{n}(t)\right)+q_{n}\left(t, y_{1}(t), \ldots, y_{n}(t)\right) \int_{0}^{\infty} k_{n}(t, s) h_{n}\left(s,\left(y_{j}(s)\right)_{j=1}^{\infty}\right) d s \mid \\
& \leq\left|f_{n}\left(t, x_{1}(t), \ldots, x_{n}(t)\right)-f_{n}\left(t, y_{1}(t), \ldots, y_{n}(t)\right)\right| \\
& +\left|q_{n}\left(t, x_{1}(t), \ldots, x_{n}(t)\right)-q_{n}\left(t, y_{1}(t), \ldots, y_{n}(t)\right)\right| \\
& \cdot\left|\int_{0}^{\infty} k_{n}(t, s) h_{n}\left(s,\left(y_{j}(s)\right)_{j=1}^{\infty}\right) d s\right|+\left|q_{n}\left(t, x_{1}(t), \ldots, x_{n}(t)\right)\right| \\
& \cdot\left|\int_{0}^{\infty} k_{n}(t, s) h_{n}\left(s,\left(x_{j}(s)\right)_{j=1}^{\infty}\right) d s-\int_{0}^{\infty} k_{n}(t, s) h_{n}\left(s,\left(y_{j}(s)\right)_{j=1}^{\infty}\right) d s\right| \\
& \leq \varphi\left(\max _{1 \leq i \leq n}\left|x_{i}(t)-y_{i}(t)\right|\right)+\varphi\left(\max _{1 \leq i \leq n}\left|x_{i}(t)-y_{i}(t)\right|\right) b_{n}\left(r_{0}\right) \int_{0}^{\infty} a_{n}(s)\left|k_{n}(t, s)\right| d s \\
& +\left(\varphi\left(\max _{1 \leq i \leq n}\left|x_{i}(t)\right|\right)+M\right)\left|\int_{0}^{\infty} k_{n}(t, s)\left[h_{n}\left(s,\left(x_{j}(s)\right)_{j=1}^{\infty}\right)-h_{n}\left(s,\left(y_{j}(s)\right)_{j=1}^{\infty}\right)\right] d s\right| .
\end{aligned}
$$

So, as a result of condition $\left(C_{3}\right)$, we can infer there exists $T>0$ such that for $t>T$, we have

$$
\begin{aligned}
& \left|G_{n}\left(\left(x_{j}\right)_{j=1}^{\infty}\right)(t)-G_{n}\left(\left(y_{j}\right)_{j=1}^{\infty}\right)(t)\right| \\
& \leq \varphi\left(\max _{1 \leq i \leq n}\left|x_{i}(t)-y_{i}(t)\right|\right)\left(1+b_{n}\left(r_{0}\right) \int_{0}^{\infty} a_{n}(s)\left|k_{n}(t, s)\right| d s\right) \\
& +\left(\varphi\left(\max _{1 \leq i \leq n}\left|x_{i}(t)\right|\right)+M\right) 2 b_{n}\left(r_{0}\right) \int_{0}^{\infty}\left|k_{n}(t, s)\right| a_{n}(s) d s \\
& \leq \varphi(\varepsilon)\left(1+b_{n}\left(r_{0}\right) Q\right)+2\left(\varphi\left(r_{0}\right)+M\right) b_{n}\left(r_{0}\right) \varepsilon .
\end{aligned}
$$

Now, we assume that $t \in[0, T]$. By applying the assumptions, we have 


$$
\begin{aligned}
& \left|G_{n}\left(\left(x_{j}\right)_{j=1}^{\infty}\right)(t)-G_{n}\left(\left(y_{j}\right)_{j=1}^{\infty}\right)(t)\right| \leq \\
& \leq \varphi\left(\max _{1 \leq i \leq n}\left|x_{i}(t)-y_{i}(t)\right|\right)\left(1+b_{n}\left(r_{0}\right) Q\right) \\
& +\left(\varphi\left(\max _{1 \leq i \leq n}\left|x_{i}(t)\right|\right)+M\right)\left|\int_{0}^{T} k_{n}(t, s)\left[h_{n}\left(s,\left(x_{j}(s)\right)_{j=1}^{\infty}\right)-h_{n}\left(s,\left(y_{j}(s)\right)_{j=1}^{\infty}\right)\right] d s\right| \\
& +\left(\varphi\left(\max _{1 \leq i \leq n}\left|x_{i}(t)\right|\right)+M\right)\left|\int_{T}^{\infty} k_{n}(t, s)\left[h_{n}\left(s,\left(x_{j}(s)\right)_{j=1}^{\infty}\right)-h_{n}\left(s,\left(y_{j}(s)\right)_{j=1}^{\infty}\right)\right] d s\right| \\
& \leq \varphi(\varepsilon)\left(1+b_{n}\left(r_{0}\right) Q\right)+\left(\varphi\left(r_{0}\right)+M\right)\left(T K_{T}^{n} \omega_{r_{0}}^{T}\left(h_{n}, \varepsilon\right)+2 b_{n}\left(r_{0}\right) \int_{T}^{\infty}\left|k_{n}(t, s)\right| a_{n}(s) d s\right),
\end{aligned}
$$

where

$$
\begin{aligned}
K_{T}^{n}= & \sup \left\{k_{n}(t, s): t, s \in[0, T]\right\} \\
\omega_{r_{0}}^{T}\left(h_{n}, \varepsilon\right)= & \sup \left\{\left|h_{n}\left(s,\left(x_{j}\right)_{j=1}^{\infty}\right)-h_{n}\left(s,\left(y_{j}\right)_{j=1}^{\infty}\right)\right|: s \in[0, T],\right. \\
& \left.x_{i}, y_{i} \in\left[-r_{0}, r_{0}\right],\left|x_{i}-y_{i}\right| \leq \varepsilon\right\} .
\end{aligned}
$$

By using the continuity of $h_{n}$ on the compact set $[0, T] \times\left[-r_{0}, r_{0}\right]^{\omega}$ ( Tychonoff's theorem implies that $\left[-r_{0}, r_{0}\right]^{\omega}$ is a compact space), we have $\omega_{r_{0}}^{T}\left(h_{n}, \varepsilon\right) \longrightarrow 0$ as $\varepsilon \longrightarrow$ 0 . Moreover, in view of assumption $\left(C_{4}\right)$ we can choose $T$ in such a way that last term of the above estimate is sufficiently small. Thus $G_{n}$ is a continuous function on $\left(\bar{B}_{r_{0}}\right)^{\omega}$. Also with similar argument and using conditions $\left(B_{1}\right)-\left(B_{2}\right)$ we have $F$ is a continuous function on $\left(\bar{B}_{r_{0}}\right)^{\omega}$. Now we show that $F_{n}$ and $G_{n}$ satisfy all the conditions of Colloraly 3. Let $X_{j}$ and $Y_{j}$ be nonempty and bounded subsets of $\bar{B}_{r_{0}}$ for all $j \in \mathbb{N}$ such that $\sup \left(\mu\left(X_{i}\right)\right)<\infty$ and $\sup \left(\mu\left(Y_{i}\right)\right)<\infty$. Assume that $T>0$ and $\varepsilon>0$ are arbitrary constants. Also we take $t_{1}, t_{2} \in[0, T]$, with $\left|t_{2}-t_{1}\right| \leq \varepsilon$ and $x_{j} \in X_{j}$ and $y_{j} \in Y_{j}$ for all $j \in \mathbb{N}$. Then we have 


$$
\begin{aligned}
& \left|F_{n}\left(\left(x_{j}\right)_{j=1}^{\infty}\right)\left(t_{2}\right)-F_{n}\left(\left(x_{j}\right)_{j=1}^{\infty}\right)\left(t_{1}\right)\right| \\
& =\mid f_{n}\left(t_{2}, x_{1}\left(t_{2}\right), \ldots x_{n}\left(t_{2}\right)\right)+q_{n}\left(t_{2}, x_{1}\left(t_{2}\right), \ldots x_{n}\left(t_{2}\right)\right) \\
& \cdot \int_{0}^{\beta_{n}\left(t_{2}\right)} g_{n}\left(t_{2}, s,\left(x_{j}(s)\right)_{j=1}^{\infty}\right) d s \\
& -f_{n}\left(t_{1}, x_{1}\left(t_{1}\right), \ldots x_{n}\left(t_{1}\right)\right)+q_{n}\left(t_{1}, x_{1}\left(t_{1}\right), \ldots x_{n}\left(t_{1}\right)\right) \\
& \cdot \int_{0}^{\beta_{n}\left(t_{1}\right)} g_{n}\left(t_{1}, s,\left(x_{j}(s)\right)_{j=1}^{\infty}\right) d s \\
& \leq\left|f_{n}\left(t_{2}, x_{1}\left(t_{2}\right), \ldots x_{n}\left(t_{2}\right)\right)-f_{n}\left(t_{1}, x_{1}\left(t_{2}\right), \ldots x_{n}\left(t_{2}\right)\right)\right| \\
& +\left|f_{n}\left(t_{1}, x_{1}\left(t_{2}\right), \ldots x_{n}\left(t_{2}\right)\right)-f_{n}\left(t_{1}, x_{1}\left(t_{1}\right), \ldots x_{n}\left(t_{1}\right)\right)\right| \\
& +\left|q_{n}\left(t_{2}, x_{1}\left(t_{2}\right), \ldots x_{n}\left(t_{2}\right)\right)-q_{n}\left(t_{1}, x_{1}\left(t_{2}\right), \ldots x_{n}\left(t_{2}\right)\right)\right| \mid \\
& \cdot \int_{0}^{\beta_{n}\left(t_{1}\right)} g_{n}\left(t_{2}, s,\left(x_{j}(s)\right)_{j=1}^{\infty}\right) d s \\
& +\left|q_{n}\left(t_{1}, x_{1}\left(t_{2}\right), \ldots x_{n}\left(t_{2}\right)\right)-q_{n}\left(t_{1}, x_{1}\left(t_{1}\right), \ldots x_{n}\left(t_{1}\right)\right)\right| \mid \\
& \cdot \int_{0}^{\beta_{n}\left(t_{2}\right)} g_{n}\left(t_{2}, s,\left(x_{j}(s)\right)_{j=1}^{\infty}\right) d s \\
& +\left|q_{n}\left(t_{1}, x_{1}\left(t_{1}\right), \ldots x_{n}\left(t_{1}\right)\right)\right| \mid \int_{0}^{\beta_{n}\left(t_{2}\right)} g_{n}\left(t_{2}, s,\left(x_{j}(s)\right)_{j=1}^{\infty}\right) d s \\
& -\int_{0}^{\beta_{n}\left(t_{1}\right)} g_{n}\left(t_{2}, s,\left(x_{j}(s)\right)_{j=1}^{\infty}\right) d s \\
& +\left|q_{n}\left(t_{1}, x_{1}\left(t_{1}\right), \ldots x_{n}\left(t_{1}\right)\right)\right| \mid \int_{0}^{\beta_{n}\left(t_{1}\right)} g_{n}\left(t_{2}, s,\left(x_{j}(s)\right)_{j=1}^{\infty}\right) d s \\
& -\int_{0}^{\beta_{n}\left(t_{1}\right)} g_{n}\left(t_{1}, s,\left(x_{j}(s)\right)_{j=1}^{\infty}\right) d s \\
& \leq \omega_{r_{0}}^{T}\left(f_{n}, \varepsilon\right)+\varphi\left(\max _{1 \leq i \leq n}\left|x_{i}\left(t_{1}\right)-x_{i}\left(t_{2}\right)\right|\right)+D \omega_{r_{0}}^{T}\left(q_{n}, \varepsilon\right) \\
& +D \varphi\left(\max _{1 \leq i \leq n}\left|x_{i}\left(t_{1}\right)-x_{i}\left(t_{2}\right)\right|\right)+\left|q_{n}\left(t_{1}, x_{1}\left(t_{1}\right), \ldots x_{n}\left(t_{1}\right)\right)\right| \mid \\
& \cdot \int_{\beta\left(t_{1}\right)}^{\beta\left(t_{2}\right)} g_{n}\left(t_{2}, s,\left(x_{j}(s)\right)_{j=1}^{\infty}\right) d s|+| q_{n}\left(t_{1}, x_{1}\left(t_{1}\right), \ldots x_{n}\left(t_{1}\right)\right)|| \\
& \cdot \int_{0}^{\beta_{n}\left(t_{1}\right)}\left[g_{n}\left(t_{2}, s,\left(x_{j}(s)\right)_{j=1}^{\infty}\right) d s-g_{n}\left(t_{1}, s,\left(x_{j}(s)\right)_{j=1}^{\infty}\right)\right] d s \mid \\
& \leq \omega_{r_{0}}^{T}\left(f_{n}, \varepsilon\right)+D \omega_{r_{0}}^{T}\left(q_{n}, \varepsilon\right)+(1+D) \varphi\left(\max _{1 \leq i \leq n} \omega^{T}\left(x_{i}, \varepsilon\right)\right) \\
& +\beta_{T} Q_{r_{0}}^{T} \omega_{r_{0}}^{T}\left(g_{n}, \varepsilon\right)+Q_{r_{0}}^{T} U_{r_{0}}^{T} \omega^{T}(\beta, \varepsilon) .
\end{aligned}
$$


Thus, we deduce

$$
\begin{aligned}
\mid F_{n}\left(\left(x_{j}\right)_{j=1}^{\infty}\right)\left(t_{2}\right) & -F_{n}\left(\left(x_{j}\right)_{j=1}^{\infty}\right)\left(t_{1}\right) \mid \leq \omega_{r_{0}}^{T}\left(f_{n}, \varepsilon\right) \\
& +D \omega_{r_{0}}^{T}\left(q_{n}, \varepsilon\right)+(1+D) \varphi\left(\max _{1 \leq i \leq n} \omega^{T}\left(x_{i}, \varepsilon\right)\right) \\
& +\beta_{T} Q_{r_{0}}^{T} \omega_{r_{0}}^{T}\left(g_{n}, \varepsilon\right)+Q_{r_{0}}^{T} U_{r_{0}}^{T} \omega^{T}(\beta, \varepsilon),
\end{aligned}
$$

and with similar argument, we get

$$
\begin{aligned}
& \left|G_{n}\left(\left(y_{j}\right)_{j=1}^{\infty}\right)\left(t_{2}\right)-G_{n}\left(\left(y_{j}\right)_{j=1}^{\infty}\right)\left(t_{1}\right)\right| \\
& \leq \omega_{r_{0}}^{T}\left(f_{n}, \varepsilon\right)+D \omega_{r_{0}}^{T}\left(q_{n}, \varepsilon\right)+\left(1+Q b\left(r_{0}\right)\right) \varphi\left(\max _{1 \leq i \leq n} \omega^{T}\left(x_{i}, \varepsilon\right)\right) \\
& +T Q_{r_{0}}^{T} V_{r_{0}}^{T} \omega_{r_{0}}^{T}\left(k_{n}, \varepsilon\right)+Q_{r_{0}}^{T} b_{n}\left(r_{0}\right) \int_{T}^{\infty}\left[\left|k_{n}\left(t_{2}, s\right)\right|+\left|k_{n}\left(t_{1}, s\right)\right|\right] a(s) d s,
\end{aligned}
$$

where

$$
\begin{aligned}
\beta_{T}= & \sup \{\beta(t): t \in[0, T]\}, \\
\omega_{r_{0}}^{T}\left(f_{n}, \varepsilon\right)= & \sup \left\{\left|f_{n}\left(t_{1}, x_{1}, \ldots, x_{n}\right)-f_{n}\left(t_{2}, x_{1}, \ldots, x_{n}\right)\right|\right. \\
& \left.: t_{1}, t_{2} \in[0, T],\left|t_{2}-t_{1}\right| \leq \varepsilon,\left|x_{i}\right| \leq r_{0}\right\}, \\
\omega_{r_{0}}^{T}\left(q_{n}, \varepsilon\right)= & \sup \left\{\left|q_{n}\left(t_{1}, x_{1}, \ldots, x_{n}\right)-q_{n}\left(t_{2}, x_{1}, \ldots, x_{n}\right)\right|\right. \\
& \left.: t_{1}, t_{2} \in[0, T],\left|t_{2}-t_{1}\right| \leq \varepsilon,\left|x_{i}\right| \leq r_{0}\right\}, \\
\omega_{r_{0}}{ }^{T}\left(g_{n}, \varepsilon\right)= & \sup \left\{\left|g_{n}\left(t_{1}, s,\left(x_{j}\right)_{j=1}^{\infty}\right)-g_{n}\left(t_{2}, s,\left(x_{j}\right)_{j=1}^{\infty}\right)\right|\right. \\
& \left.: t_{1}, t_{2}, s \in[0, T],\left|t_{2}-t_{1}\right| \leq \varepsilon, s \in\left[0, \beta_{T}\right],\left|x_{j}\right| \leq r_{0}\right\}, \\
\omega^{T}(\beta, \varepsilon)= & \sup \{|\beta(t)-\beta(s)|: t, s \in[0, T],|t-s| \leq \varepsilon\}, \\
U_{r_{0}}^{T}= & \sup \left\{\left|g_{n}\left(t, s,\left(x_{j}\right)_{j=1}^{\infty}\right)\right|: t \in[0, T], s \in\left[0, \beta_{T}\right], x_{i} \in\left[-r_{0}, r_{0}\right]\right\}, \\
Q_{r_{0}}^{T}= & \sup \left\{\left|q_{n}\left(t, x_{1}, \ldots, x_{n}\right)\right|: t \in[0, T], x_{i} \in\left[-r_{0}, r_{0}\right]\right\}, \\
\omega_{r_{0}}^{T}\left(k_{n}, \varepsilon\right)= & \sup \left\{\left|k_{n}\left(t_{1}, s\right)-k_{n}\left(t_{2}, s\right)\right|: t_{1}, t_{2}, s \in[0, T],\left|t_{2}-t_{1}\right| \leq \varepsilon\right\}, \\
V_{r_{0}}^{T}= & \sup \left\{\left|h_{n}\left(s,\left(x_{j}\right)_{j=1}^{\infty}\right)\right|: s \in[0, T], x_{i} \in\left[-r_{0}, r_{0}\right]\right\} .
\end{aligned}
$$

Since $x_{i}$ is an arbitrary element of $X_{i}$ and $y_{i}$ was an arbitrary element of $Y_{i}$ for all $i \in \mathbb{N}$ in (4.3) and (4.4), we obtain

$$
\begin{gathered}
\omega^{T}\left(F_{n}\left(\prod_{i=1}^{\infty} X_{i}\right) \leq \omega_{r_{0}}^{T}\left(f_{n}, \varepsilon\right)+D \omega_{r_{0}}^{T}\left(q_{n}, \varepsilon\right)+(1+D) \varphi\left(\max _{1 \leq i \leq n} \omega^{T}\left(X_{i}, \varepsilon\right)\right)\right. \\
+\beta_{T} Q_{r_{0}}^{T} \omega_{r_{0}}^{T}\left(g_{n}, \varepsilon\right)+Q_{r_{0}}^{T} U_{r_{0}}^{T} \omega^{T}(\beta, \varepsilon), \\
\omega^{T}\left(G_{n}\left(\prod_{i=1}^{\infty} Y_{i}\right) \leq \omega_{r_{0}}^{T}\left(f_{n}, \varepsilon\right)+D \omega_{r_{0}}^{T}\left(q_{n}, \varepsilon\right)+\left(1+Q b_{n}\left(r_{0}\right)\right) \varphi\left(\max _{1 \leq i \leq n} \omega^{T}\left(X_{i}, \varepsilon\right)\right)\right. \\
+T Q_{r_{0}}^{T} V_{r_{0}}^{T} \omega_{r_{0}}^{T}\left(k_{n}, \varepsilon\right)+Q_{r_{0}}^{T} b_{n}\left(r_{0}\right) \int_{T}^{\infty}\left[\left|k_{n}\left(t_{2}, s\right)\right|+\left|k_{n}\left(t_{1}, s\right)\right|\right] a(s) d s,
\end{gathered}
$$


and by the uniform continuity of $f_{n}, q_{n}, g_{n}$ and $\beta$ on the compact sets $[0, T] \times$ $\left[-r_{0}, r_{0}\right]^{n},[0, T] \times\left[-r_{0}, r_{0}\right]^{n},[0, T] \times\left[0, \beta_{T}\right] \times\left[-r_{0}, r_{0}\right]^{\omega}$ and $[0, T]$ respectively, we have $\omega_{r_{0}}{ }^{T}\left(f_{n}, \varepsilon\right) \longrightarrow 0, \omega_{r_{0}}{ }^{T}\left(q_{n}, \varepsilon\right) \longrightarrow 0, \omega_{r_{0}}{ }^{T}\left(g_{n}, \varepsilon\right) \longrightarrow 0$ and $\omega^{T}(\beta, \varepsilon) \longrightarrow 0$ as $\varepsilon \longrightarrow 0$. Therefore we obtain

$$
\begin{gathered}
\omega_{0}^{T}\left(F_{n}\left(\prod_{i=1}^{\infty} X_{i}\right)\right) \leq(1+D) \varphi\left(\max _{1 \leq i \leq n} \omega_{0}^{T}\left(X_{i}\right)\right), \\
\omega_{0}^{T}\left(G_{n}\left(\prod_{i=1}^{\infty} Y_{i}\right) \leq\right. \\
\left(1+Q b_{n}\left(r_{0}\right)\right) \varphi\left(\max _{1 \leq i \leq n} \omega_{0}^{T}\left(Y_{i}, \varepsilon\right)\right) \\
+Q_{r_{0}}^{T} b_{n}\left(r_{0}\right) \int_{T}^{\infty}\left[\left|k_{n}\left(t_{2}, s\right)\right|+\left|k_{n}\left(t_{1}, s\right)\right|\right] a(s) d s .
\end{gathered}
$$

Now taking $T \longrightarrow \infty$ and by using of assumption $\left(C_{4}\right)$, we get

$$
\omega_{0}\left(F_{n}\left(\prod_{i=1}^{\infty} X_{i}\right)\right) \leq(1+D) \varphi\left(\max _{1 \leq i \leq n} \omega_{0}\left(X_{i}\right)\right),
$$

and

$$
\omega_{0}\left(G_{n}\left(\prod_{i=1}^{\infty} Y_{i}\right)\right) \leq\left(1+Q b_{n}\left(r_{0}\right)\right) \varphi\left(\max _{1 \leq i \leq n} \omega_{0}\left(Y_{i}\right)\right) .
$$

On the other hand, for all $x_{i}, u_{i} \in X_{i}, y_{i}, v_{i} \in Y_{i}(i \in \mathbb{N})$ and $t \in \mathbb{R}_{+}$, we get

$$
\begin{aligned}
& \left|F_{n}\left(\left(x_{j}\right)_{j=1}^{\infty}\right)(t)-F_{n}\left(\left(u_{j}\right)_{j=1}^{\infty}\right)(t)\right| \leq(1+D) \varphi\left(\max _{1 \leq i \leq n}\left|x_{i}(t)-u_{i}(t)\right|\right) \\
& +\left(\varphi\left(r_{0}\right)+M\right)\left|\int_{0}^{\beta(t)}\right| g_{n}\left(t, s,\left(x_{j}(s)\right)_{j=1}^{\infty}\right)-g_{n}\left(t, s,\left(u_{j}(s)\right)_{j=1}^{\infty}\right) \mid d s,
\end{aligned}
$$

and with similar argument

$$
\begin{aligned}
& \left|G_{n}\left(\left(y_{j}\right)_{j=1}^{\infty}\right)(t)-G_{n}\left(\left(v_{j}\right)_{j=1}^{\infty}\right)(t)\right| \leq\left(1+Q b_{n}\left(r_{0}\right)\right) \varphi\left(\max _{1 \leq i \leq n}\left|y_{i}(t)-v_{i}(t)\right|\right) \\
& +\left(\varphi\left(r_{0}\right)+M\right) \mid \int_{0}^{\infty} k_{n}(t, s)\left[h_{n}\left(t, s,\left(y_{j}(s)\right)_{j=1}^{\infty}\right)-h_{n}\left(t, s,\left(v_{j}(s)\right)_{j=1}^{\infty}\right)\right] d s \\
\leq & \left(1+Q b_{n}\left(r_{0}\right)\right) \varphi\left(\max _{1 \leq i \leq n}\left|y_{i}(t)-v_{i}(t)\right|\right) \\
& +\left(\varphi\left(r_{0}\right)+M\right) 2 b_{n}\left(r_{0}\right) \int_{0}^{\infty}\left|k_{n}(t, s)\right| a(s) d s .
\end{aligned}
$$

Thus

$$
\begin{aligned}
& \operatorname{diam}\left(F_{n}\left(\prod_{i=1}^{\infty} X_{i}\right)(t)\right) \leq(1+D) \varphi\left(\max _{1 \leq i \leq n} \operatorname{diam}\left(X_{i}(t)\right)\right) \\
& +\left(\varphi\left(r_{0}\right)+M\right) \mid \int_{0}^{\beta(t)}\left[g_{n}\left(t, s,\left(x_{j}(s)\right)_{j=1}^{\infty}\right)-g_{n}\left(t, s,\left(u_{j}(s)\right)_{j=1}^{\infty}\right)\right] d s
\end{aligned}
$$


and

$$
\begin{aligned}
& \operatorname{diam}\left(G_{n}\left(\prod_{i=1}^{\infty} Y_{i}\right)(t)\right) \leq\left(1+Q b_{n}\left(r_{0}\right)\right) \varphi\left(\max _{1 \leq i \leq n} \operatorname{diam}\left(Y_{i}(t)\right)\right) \\
& +\left(\varphi\left(r_{0}\right)+M\right) 2 b_{n}\left(r_{0}\right) \int_{0}^{\infty}\left|k_{n}(t, s)\right| a(s) d s .
\end{aligned}
$$

If take $t \longrightarrow \infty$ in the inequalities (4.7) and (4.8), then using $\left(B_{2}\right)$ and $\left(C_{3}\right)$, we have

$$
\limsup _{t \rightarrow \infty} \operatorname{diam}_{n}\left(\prod_{i=1}^{\infty} X_{i}\right)(t) \leq(1+D) \varphi\left(\max _{1 \leq i \leq n} \limsup _{t \longrightarrow \infty} \operatorname{diam}\left(X_{i}(t)\right)\right),
$$

and

$$
\underset{t \rightarrow \infty}{\limsup } \operatorname{diam} G_{n}\left(\prod_{i=1}^{\infty} Y_{i}\right)(t) \leq\left(1+Q b_{n}\left(r_{0}\right)\right) \varphi\left(\max _{1 \leq i \leq n} \limsup _{t \rightarrow \infty} \operatorname{diam}\left(X_{i}(t)\right)\right) .
$$

Further, combining (4.5) and (4.9), we get

$$
\begin{aligned}
\underset{t \rightarrow \infty}{\limsup \operatorname{diam}_{n}\left(\prod_{i=1}^{\infty} X_{i}\right)(t)+\omega_{0}\left(F_{n}\left(\prod_{i=1}^{\infty} X_{i}\right)\right) \leq(1+D)} \\
{\left[\varphi\left(\max _{1 \leq i \leq n} \omega_{0}\left(X_{i}\right)\right)+\varphi\left(\max _{1 \leq i \leq n} \limsup _{t \rightarrow \infty} \operatorname{diam}\left(X_{i}(t)\right)\right)\right], }
\end{aligned}
$$

from (4.6) and (4.10) we have

$$
\begin{aligned}
\limsup _{t \rightarrow \infty} \operatorname{diam}_{n}\left(\prod_{i=1}^{\infty} Y_{i}\right)(t)+\omega_{0}\left(G_{n}\left(\prod_{i=1}^{\infty} Y_{i}\right)\right) \leq & \left(1+Q b_{n}\left(r_{0}\right)\right)\left[\varphi\left(\max _{1 \leq i \leq n} \omega_{0}\left(Y_{i}\right)\right)\right. \\
& \left.+\varphi\left(\max _{1 \leq i \leq n} \limsup _{t \rightarrow \infty} \operatorname{diam}\left(Y_{i}(t)\right)\right)\right] .
\end{aligned}
$$

Since $\varphi$ is concave, (4.11) and (4.12) imply

$$
\frac{1}{4} \mu\left(F_{n}\left(\prod_{i=1}^{\infty} X_{i}\right)\right)+\frac{1}{4} \mu\left(G_{n}\left(\prod_{i=1}^{\infty} Y_{i}\right)\right) \leq \varphi^{\prime}\left(\frac{1}{4} \sup _{i} \mu\left(X_{i}\right)+\frac{1}{4} \sup _{i} \mu\left(Y_{i}\right)\right),
$$

where $\varphi^{\prime}(t)=\left(\max \left\{1+D, \sup _{n \in \mathbb{N}}\left\{1+Q b_{n}\left(r_{0}\right)\right\}\right\}\right) \varphi(t)$. Taking $\mu^{\prime}=\frac{1}{4} \mu$ and $\psi(t)=t$.

Then we get

$$
\mu^{\prime}\left(F_{n}\left(\prod_{i=1}^{\infty} X_{i}\right)\right)+\mu^{\prime}\left(G_{n}\left(\prod_{i=1}^{\infty} Y_{i}\right)\right) \leq \varphi^{\prime}\left(\sup _{i}\left\{\mu^{\prime}\left(X_{i}\right)+\mu^{\prime}\left(Y_{i}\right)\right\}\right) .
$$

Now by using Colloraly 3 , there exist $\left(x_{i}\right)_{i=1}^{\infty},\left(y_{i}\right)_{i=1}^{\infty} \in\left(B C\left(\mathbb{R}_{+}\right)\right)^{\omega}$ such that

$$
\begin{aligned}
x_{n}(t)= & f_{n}\left(t, x_{1}(t), \ldots, x_{n}(t)\right) \\
& +q_{n}\left(t, x_{1}(t), \ldots, x_{n}(t)\right) \int_{0}^{\beta_{n}(t)} g_{n}\left(t, s,\left(x_{j}(s)\right)_{j=1}^{\infty}\right) d s,
\end{aligned}
$$


and

$$
\begin{aligned}
y_{n}(t)= & f_{n}\left(t, y_{1}(t), \ldots, y_{n}(t)\right) \\
& \left.+q_{n}\left(t, y_{1}(t), \ldots, y_{n}(t)\right) \int_{0}^{\infty} k_{n}(t, s) h_{n}\left(s,\left(y_{j}(s)\right)_{j=1}^{\infty}\right) d s\right),
\end{aligned}
$$

and this completes the proof.

Example 2. Consider the following system of functional integral equations

$$
x_{n}(t)=\frac{1}{4} \arctan \left(\frac{1}{n} \sum_{i=1}^{n}\left|x_{i}(t)\right|\right)+\int_{0}^{\frac{\sin t}{n}} \frac{s \cos \left(x_{n}\left(s^{2}\right)\right)}{e^{t^{2}}} d s,
$$

and

$$
y_{n}(t)=\frac{1}{4} \arctan \left(\frac{1}{n} \sum_{i=1}^{n}\left|y_{i}(t)\right|\right)+\int_{0}^{\infty} \frac{t\left(e^{-n s}-1\right)}{\left(t^{2}+1\right) n} \sum_{i=1}^{\infty} \sqrt[-i s-1]{y_{i}(s)} d s .
$$

Eq. (4.13) is a special case of Eq. (1.1) and Eq. (4.14) is a special case of Eq. (1.2) where

$$
\begin{aligned}
& f_{n}\left(t, x_{1}, \ldots, x_{n}\right)=\frac{1}{4} \arctan \left(\frac{1}{n} \sum_{i=1}^{n}\left|x_{i}\right|\right), \\
& q_{n}\left(t, x_{1}, \ldots, x_{n}\right)=1, \\
& g_{n}\left(t, s,\left(x_{j}\right)_{j=1}^{\infty}\right)=\frac{s \cos x_{n}}{e^{t^{2}}}, \\
& k_{n}(t, s)=\frac{t}{\left(t^{2}+1\right) n}\left(e^{-n s}-1\right), \\
& h_{n}\left(s,\left(y_{j}\right)_{j=1}^{\infty}\right)=\sum_{i=1}^{\infty} e^{-i s \sqrt[2 n-1]{y_{i}},} \\
& a_{n}(s)=\frac{1}{e^{s}-1}, b_{n}(r)=\sqrt[2 n-1]{r}, \beta_{n}(t)=\frac{\sin t}{n} .
\end{aligned}
$$

Suppose that $t \in \mathbb{R}_{+}$and $\left|x_{i}\right| \geq\left|y_{i}\right|$. Now, by taking $\varphi(t)=\frac{1}{4} \arctan (t)$ we have

$$
\begin{aligned}
\left|f_{n}\left(t, x_{1}, \ldots, x_{n}\right)-f_{n}\left(t, y_{1}, \ldots, y_{n}\right)\right| & \leq \frac{1}{4}\left|\arctan \left(\frac{1}{n} \sum_{i=1}^{n}\left|x_{i}\right|\right)-\arctan \left(\frac{1}{n} \sum_{i=1}^{n}\left|y_{i}\right|\right)\right| \\
& \leq \frac{1}{4} \arctan \left(\frac{1}{n} \sum_{i=1}^{n}\left|x_{i}-y_{i}\right|\right) \\
& \leq \frac{1}{4} \arctan \left(\max _{1 \leq i \leq n}\left|x_{i}-y_{i}\right|\right) \\
& =\varphi\left(\max _{1 \leq i \leq n}\left|x_{i}-y_{i}\right|\right) .
\end{aligned}
$$

The case $\left|y_{i}\right| \geq\left|x_{i}\right|$ can be treated in the same way. Moreover,

$$
M:=\sup \left\{\max \left\{\left|f_{n}(t, 0, \ldots, 0)\right|,\left|q_{n}(t, 0, \ldots, 0)\right|\right\}: t \in \mathbb{R}_{+}, n \in \mathbb{N}\right\}=1<\infty .
$$


Thus from (4.15) we infer that condition $\left(\mathrm{A}_{1}\right)$ holds. The condition $\left(\mathrm{B}_{1}\right)$ is obvious. Also, $g_{n}$ is continuous and

$$
\begin{gathered}
D=\sup \left\{\left|\int_{0}^{\beta_{n}(t)} g_{n}\left(t, s,\left(x_{j}(s)\right)_{j=1}^{\infty}\right) d s\right|: t \in \mathbb{R}_{+}, x_{j} \in B C\left(\mathbb{R}_{+}\right), 1 \leq n<\infty\right\} \\
=\sup \left\{\left|\int_{0}^{\frac{\sin t}{n}} \frac{s \cos \left(x_{n}\left(s^{2}\right)\right)}{e^{t^{2}}} d s\right|: t \in \mathbb{R}_{+}, x_{j} \in B C\left(\mathbb{R}_{+}\right), 1 \leq n<\infty\right\}=\frac{1}{2}, \\
\left.\lim _{t \rightarrow \infty} \mid \int_{0}^{\frac{\sin t}{n}} \frac{s \cos \left(x_{n}\left(s^{2}\right)\right)}{e^{t^{2}}}-\frac{s \cos \left(y_{n}\left(s^{2}\right)\right)}{e^{t^{2}}}\right) d s \mid=0,
\end{gathered}
$$

uniformly respect to $x_{j}, y_{j} \in B C\left(\mathbb{R}_{+}\right)$, which implies that condition $\left(\mathrm{B}_{2}\right)$ is satisfied. The condition $\left(\mathrm{C}_{1}\right)$ clearly is evident. In order to show that condition $\left(\mathrm{C}_{2}\right)$ is satisfied, let us assume that $\sup _{1<i<\infty}\left|y_{i}\right|<\infty$, so we have

$$
\begin{aligned}
\left|h_{n}\left(t,\left(y_{j}\right)_{j=1}^{\infty}\right)\right|=\sum_{i=1}^{\infty} e^{-i t} \sqrt[2 n-1]{y_{i}} & \leq \sum_{i=1}^{\infty} e^{-i t} \sqrt[2 n-1]{\sup _{1 \leq i<\infty}\left|y_{i}\right|} \\
& \leq \frac{1}{e^{t}-1} \sqrt[2 n-1]{\sup _{1 \leq i<\infty}\left|y_{i}\right|} \\
& =a_{n}(t) b_{n}\left(\sup _{1 \leq i<\infty}\left|y_{i}\right|\right) .
\end{aligned}
$$

On the other hand, the function $s \longrightarrow a_{n}(s) k_{n}(t, s)$ is integrable over $\mathbb{R}_{+}$for any fixed $t \in \mathbb{R}_{+}$and $n \in \mathbb{N}$. Thus, condition $\left(\mathrm{C}_{2}\right)$ is valid. Further, we get:

$$
\begin{aligned}
Q & =\sup \left\{\int_{0}^{\infty} a_{n}(s)\left|k_{n}(t, s)\right| d s: t \in \mathbb{R}_{+}, n \in \mathbb{N}\right\} \\
& =\sup \left\{\frac{t}{\left(t^{2}+1\right) n} \int_{0}^{\infty} \frac{e^{-n s}-1}{e^{s}-1} d s: t \in \mathbb{R}_{+}, n \in \mathbb{N}\right\} \\
& =\sup \left\{\frac{t}{\left(t^{2}+1\right) n} n: t \in \mathbb{R}_{+}, n \in \mathbb{N}\right\}=\frac{1}{2}<\infty,
\end{aligned}
$$

and

$$
\lim _{t \longrightarrow \infty} \int_{0}^{\infty} a_{n}(s)\left|k_{n}(t, s)\right| d s=\lim _{t \longrightarrow \infty} \frac{t}{\left(t^{2}+1\right) n} \int_{0}^{\infty} \frac{e^{-n s}-1}{e^{s}-1} d s=\lim _{t \longrightarrow \infty} \frac{t}{t^{2}+1}=0 .
$$

This impliese that the condition $\left(\mathrm{C}_{3}\right)$ holds. Moreover, for arbitrarily fixed $T>0$ we obtain:

$$
\begin{aligned}
\int_{T}^{\infty} a_{n}(s)\left|k_{n}(t, s)\right| d s & =\frac{t}{\left(t^{2}+1\right) n} \int_{T}^{\infty} \frac{e^{-n s}-1}{e^{s}-1} d s \\
& \leq \frac{1}{2 n}\left[e^{-n T}+e^{-(n-1) T}+e^{-(n-2) T}+\ldots+e^{-T}\right] .
\end{aligned}
$$


From the above estimate, we infer that condition $\left(\mathrm{C}_{4}\right)$ holds. It is easy to see that each number $r \geq 4$ satisfies the inequality in condition $\left(\mathrm{D}_{1}\right)$, i.e.,

$$
(1+D)(\varphi(r)+M)=\left(1+\frac{1}{2}\right)\left(\frac{1}{4} \arctan (r)+1\right) \leq r,
$$

and

$$
\left(1+Q b_{n}(r)\right)(\varphi(r)+M)=\left(1+\frac{1}{2} \sqrt[2 n-1]{r}\right)\left(\frac{1}{4} \arctan (r)+1\right) \leq r,
$$

for all $n \in \mathbb{N}$ such that

$$
\left(\max \left\{1+D, \sup _{n \in \mathbb{N}}\left\{1+Q b_{n}\left(r_{0}\right)\right\}\right\}\right) \varphi(t)=\left(\max \left\{1+\frac{1}{2}, \sup _{n \in \mathbb{N}}\left\{1+\frac{1}{2} \sqrt[2 n-1]{4}\right\}\right\}\right) \frac{1}{4} \arctan (t)<t .
$$

Thus, we can take $r_{0}=4$. Consequently, all the conditions of Theorem 2 are satisfied. Hence the system of functional integral equations (4.13) and (4.14) have at least one solution put which belongs to the space $\left(B C\left(\mathbb{R}_{+}\right)\right)^{\omega}$.

\section{REFERENCES}

[1] R. P. Agarwal, M. Meehan, and D. O'Regan, Fixed point theory and applications. Cambridge university press, 2001, vol. 141.

[2] A. Aghajani, R. Allahyari, and M. Mursaleen, "A generalization of darbo's theorem with application to the solvability of systems of integral equations," J. Comput. Appl. Math., vol. 260, pp. 68-77, 2014, doi: 10.1016/j.cam.2013.09.039.

[3] A. Aghajani, J. Banaś, and N. Sabzali, "Some generalizations of darbo fixed point theorem and applications," Bull. Belg. Math. Soc. Simon Stevin, vol. 20, pp. 345-358, 2013, doi: 10.36045/bbms/1369316549.

[4] A. Aghajani and Y. Jalilian, "Existence of nondecreasing positive solutions for a system of sigular integral equations," Mediter. J. Math., no. 8, pp. 563-576, 2011, doi: 10.1007/s00009-010-0095-3.

[5] A. Aghajani and N. Sabzali, "Existence of coupled fixed points via measure of noncompactness and applications," J. Nonlinear Convex A., vol. 15, no. 5, pp. 941-952, 2014.

[6] R. Arab, "The existence of fixed points via the measure of non-compactness and its application to functional integral equations," Mediterr. J. Math., vol. 13, 2015, doi: 10.1007/s00009-014-0506.

[7] R. Arab, "Some fixed point theorems in generalized darbo fixed point theorem and the existence of solutions for system of integral equations," J. Korean Math. Soc., vol. 52, no. 1, pp. 125-139, 2015, doi: 10.4134/JKMS.2015.52.1.125.

[8] R. Arab, "Some generalizations of darbo fixed point theorem and its application," Miskolc Math. Notes., vol. 18, no. 2, pp. 595-610, 2017, doi: 10.18514/MMN.2017.1202.

[9] R. Arab, R. Allahyari, and A. S. Haghighi, "Existence of solutions of infinite systems of integral equations in two variables via measure of noncompactness," Appl. Math. Comput., vol. 246, pp. 283-291, 2014, doi: 10.1016/j.amc.2014.08.023.

[10] J. Banaś, "Measures of noncompactness in the space of continuous tempered functions," Demonstratio Math, vol. 14, pp. 127-133, 1981, doi: 10.1515/dema-1981-0110.

[11] J. Banaś and K. Goebel, Measures of noncompactness in Banach spaces. Marcel Dekker, Inc, 1980, vol. 60.

[12] J. Banaś and L. Millenia, "Solvability of infinite systems of differential equations in banach sequence spaces," J. Comput. Appl. Math., vol. 137, pp. 363-375, 2001, doi: 10.1016/S03770427(00)00708-1.

[13] J. B. Conway, A Course in Functional Analysis, 1994. 
[14] K. Deimling, Ordinary Differential Equations in Banach Spaces. Springer Verlag, Berlin, 1977, vol. 596.

[15] E. Hille, "Pathology of infinite systems of linear first order differential equations with constant coefficients," Ann. Mat. Pura Appl., vol. 55, pp. 135-144, 1961, doi: 10.1007/BF02412080.

[16] M. Mursaleen, "Application of measure of noncompactness to infinite systems of differential equations," Canad. Math. Bull., vol. 56, no. 2, pp. 388-394, 2013, doi: 10.4153/CMB-2011-170-7.

[17] M. Mursaleen and A. Alotaibi, "Infinite system of differential equations in some bk spaces," Abstr. Appl. Anal., vol. 2012, 2012, doi: 10.1155/2012/863483.

[18] M. Oguztorreli, "On the neural equations of cowan and stein," Util. Math., vol. 2, pp. 305-315, 1972.

[19] L. Olszowy, "Solvability of infinite systems of singular integral equations in fréchet space of continuous functions," Comput. Math. Appl., vol. 59, no. 8, pp. 2794-2801, 2010, doi: 10.1016/j.camwa.2010.01.049.

[20] K. Persidskii, "Countable systems of differential equations and stability of their solutions iii: Fundamental theorems on stability of solutions of countable many differential equations," Izv. Akad. Nauk Kazach., vol. 9, pp. 11-34, 1961

[21] R. Rzepka and K. Sadarangani, "On solutions of an infinite system of singular integral equations," Math. Comput. Modelling, vol. 45, pp. 1265-1271, 2007, doi: 10.1016/j.mcm.2006.11.006.

[22] O. Zautykov, "Countable systems of differential equations and their applications," Differ. Uravn., vol. 1, pp. 162-170, 1965.

[23] O. Zautykov and K. Valeev, "Infinite systems of differential equations," Izdat. Nauka Kazach., 1974.

Authors' addresses

Reza Allahyari

Department of Mathematics, Mashhad Branch, Islamic Azad University, Mashhad, Iran.

E-mail address: rezaallahyariemshdiau.ac.ir

\section{Reza Arab}

Corresponding author, Department of Mathematics, Sari Branch, Islamic Azad University, Sari, Iran.

E-mail address: mathreza.arabeiausari.ac.ir

Ali Shole Haghighi

Department of Mathematics, Mashhad Branch, Islamic Azad University, Mashhad, Iran.

E-mail address: ali.sholehaghighi@gmail.com 